\title{
4-phenylbutyric acid alleviates bleomycin-induced pulmonary fibrosis in mouse via inhibition of endoplasmic reticulum stress
}

\section{Yun Yang}

Changzhou First People's Hospital

Deyue Cui

Changzhou First People's Hospital

Hui Bi

Changzhou First People's Hospital

Qian He

Changzhou First People's Hospital

\section{Yunqi Ge}

Changzhou First People's Hospital

\section{Qiudi Zhang}

Changzhou First People's Hospital

\section{Jun Zhou}

Changzhou First People's Hospital

Hui Qiu ( $\square$ naturehui@126.com )

Changzhou First People's Hospital https://orcid.org/0000-0002-4137-889X

\section{Research article}

Keywords: 4-phenylbutyric acid, endoplasmic reticulum stress, pulmonary fibrosis, ATF6, CHOP

Posted Date: June 9th, 2020

DOI: https://doi.org/10.21203/rs.3.rs-29721/v1

License: (9) (1) This work is licensed under a Creative Commons Attribution 4.0 International License. Read Full License 


\section{Abstract}

Background: 4-phenylbutyric acid (4-PBA) is a chemical chaperone that may aid the folding of proteins and alleviate endoplasmic reticulum (ER) stress by inhibiting the unfolded protein response (UPR). This study explores the effects of 4-PBA on idiopathic pulmonary fibrosis (IPF) using a murine model of bleomycin (BLM)-induced pulmonary fibrosis.

Methods: Pulmonary fibrosis was induced in C57BL/ 6 mice by intratracheal injection of BLM. Sixty mice were randomly allocated into three groups: BLM group $(n=20), B L M+4-P B A$ group $(n=20)$, and control group $(n=20)$. Lung tissues and lung function were analyzed to evaluate the degree of pulmonary fibrosis and the survival of the mice was noted. The expression levels of the ER stress markers activating transcription factor 6 (ATF6) and C/EBP Homologous Protein (CHOP) were analyzed in lung tissues from IPF patients and healthy controls as well as the mice.

Results: Lung tissues from IPF patients expressed significantly higher levels of ATF6 and CHOP compared to those from healthy controls. BLM induced significant collagen deposition in the lungs of the mice, which was prevented by 4-PBA. 4-PBA also dramatically improved pulmonary function and increased the survival rate in the BLM+4-PBA group compared to that in the BLM group. Both the protein and mRNA expression levels of ATF6 and CHOP were significantly reduced in mouse lung tissue after 2 weeks of 4-PBA treatment.

Conclusions: This study demonstrated that 4-PBA treatment could alleviate BLM-induced pulmonary fibrosis in mice via the attenuation of ER stress.

\section{Background}

Idiopathic pulmonary fibrosis (IPF) is a lung disease of unknown etiology characterized by chronic, progressive, and diffuse parenchymal pathology. Despite decades of research, there is still no effective treatment available for IPF and the underlying pathogenesis is poorly understood. Accumulating evidence suggests that repetitive injury to alveolar epithelial cells activate the differentiation of pulmonary fibroblasts to myofibroblasts, causing extracellular matrix deposition and lung remodeling [1, 2]. Endoplasmic reticulum (ER) stress is emerging as a vital player in alveolar epithelial cell apoptosis and the proliferation of profibrotic cells in lung tissues during the course of IPF [3].

The ER is the largest organelle in cells and plays a critical role in many cellular processes, including protein folding and transport [4]. When the inner stability of the ER is disrupted by extracellular stimuli and changes in intracellular homeostasis, the unfolded protein response (UPR) may be activated due to the accumulation of misfolded and unfolded proteins in the ER lumen. If the UPR fails to restore ER homeostasis, ER stress occurs, disrupting the protein folding process in the ER [5].

Three major signaling pathways are involved in ER stress in mammalian cells: inositol-requiring enzyme 1 alpha (IRE1 a), PKR-like ER kinase (PERK), and activating transcription factor 6 (ATF6) pathways. Under 
normal conditions, IRE1, PERK, and ATF6 are bound by chaperone proteins such as immunoglobulin heavy-chain-binding protein (BiP; also known as GRP78) in an inactive state. Chaperone proteins assist in the normal folding of proteins. Owning to the higher affinity of BiP for misfolded proteins, BiP dissociates from the three ER sensors as misfolded proteins accumulate in the ER lumen, which initiates signaling cascades to protect and restore the function of the ER [6]. However, severe or prolonged ER stress instead activates the downstream cell death pathways through caspase-12, C/EBP homologous protein (CHOP), and c-Jun NH2-teiminal kinase (JNK) [7].

ER stress and UPR have been found to be involved in a number of human diseases such as cardiac fibrosis, diabetes, some neurological diseases, and also IPF [8-10]. The potential link between ER stress and pulmonary fibrosis was first suspected after studying a family with familial interstitial pneumonia (FIP) and a mutation in surfactant protein C (SFTPC) [11]. Subsequently, Baek et al. [12] identified the high expression of UPR markers such as CHOP and ATF6 in the alveolar epithelium of patients with sporadic IPF and FIP, even when SFTPC mutations are absent. In addition, murine models have revealed enhanced lung fibrosis following BLM treatment if the alveolar epithelium was subjected to ER stress. The underlying mechanism was proposed to be, at least in part, increased apoptosis of alveolar epithelial cells [2]. Recently, Xu et al. [13] found that particulate matter with a diameter $\leq 2.5 \mathrm{~mm}\left(\mathrm{PM}_{2.5}\right)$ could stimulate ER stress and thereby enhance BLM-induced pulmonary fibrosis in rats. Our previous studies $[14,15]$ revealed that the ER stress markers, ATF6 and CHOP, were strongly associated with the development and progression of IPF.

The chemical chaperone 4-Phenylbutyric acid (4-PBA) can act as an ammonia scavenger, a weak histone deacetylase inhibitor, and also an ER stress inhibitor [16-19]. Recent research has demonstrated that 4PBA can modulate UPR activation [20]. 4-PBA has been shown to have protective effects on various diseases such as myocardial hypertrophy, acute kidney injury, and cardiac fibrosis $[9,18,19]$. However, the use of 4-PBA in IPF has not been extensively investigated. The present study aimed to explore the effects of 4-PBA in a BLM-induced murine model of pulmonary fibrosis, which may lead to a better understanding of the pathogenesis of IPF and ultimately provide a novel treatment strategy for IPF.

\section{Methods}

Immunohistology of human lung tissues

Human lung tissues were kindly provided by Prof. Hui-ping Li (Shanghai Pulmonary Hospital, Tongji University). Lung tissues from IPF patients undergoing lung transplantation were collected. Unused healthy donor lungs served as the controls. Written informed consent was obtained from all study participants. The study was approved by the Ethics Committee of Shanghai Pulmonary Hospital (Approval No: 2014FK04, Approved date: February 25 ${ }^{\text {th }}, 2014$ ).

Lung tissue was fixed in formalin and embedded in paraffin. The expression levels of ATF6 and CHOP protein were visualized by immunohistochemistry. Positive expression was shown as a dark brown color. 
The sections were counterstained with hematoxylin and eosin. The percentages of ATF6- and CHOPpositive cells were examined under a light microscope (20x, LEICA SCN400).

Animal model and 4-PBA treatment

Specific pathogen-free grade wild-type male C57BL/6 mice (Shanghai SLAC Laboratory Animal Co. Ltd., Shanghai, China) were housed for 1-week acclimation under a 12h light/ $12 \mathrm{~h}$ dark cycle, controlled temperature $\left(22-24^{\circ} \mathrm{C}\right)$ and humidity $(50-60 \%)$ with free access to water and standard rodent chaw (normal diets with $4 \%$ of energy from lipid, XieTong Organism, China). After 1-week acclimation, mice were randomly divided into three groups: BLM group, BLM+4-PBA group, and control group, with 20 mice in each group. The study protocol was approved by the Institutional Animal Care and Use Committee at Soochow University (Approval No.: K18-028).

On day 0 , all mice were injected intraperitoneally with sodium pentobarbital $(150 \mathrm{mg} / \mathrm{kg}$ body weight solution in saline, Bio-Light Biotech Co. Ltd., Shanghai, China). Following anesthetization, mice in both the BLM group and BLM+4-PBA group received intratracheal injections of BLM (5.0 U/kg body weight solution in saline, Nippon Kayaku Co. Ltd., Tokyo, Japan) to induce lung fibrosis, while mice in the control group received equivalent volumes of vehicle (saline). In accordance with previous protocols [21], the mice were injected intraperitoneally with 4-PBA ( $500 \mathrm{mg} / \mathrm{kg}$ body weight solution in PBS) daily starting from day 15 for 2 weeks. Mice in the BLM and control groups were injected with equivalent volumes of saline. The mice were followed up for 4 weeks and their survival until day 28 was recorded. The workflow is shown in Figure 1.

Mouse lung function

The mice underwent tracheostomies and were intubated with an $18 \mathrm{G}$ intravenous catheter under sodium pentobarbital anesthesia (intraperitoneal injection). The mice were then placed in a Pulmonary Maneuvers System body plethysmograph (DSI's Buxco Electronics, Minnesota, USA) [22]. Lung function measurements were performed according to the manufacturer's instructions as previously described. For each animal, the average of three acceptable measurements was used. The parameters that were of main interest were forced vital volume (FVC), forced expiratory volume in 50 milliseconds (FEV50), and dynamic compliance (Cdyn).

Lung Collagen Measurements

Total collagen content in mice lung tissue (30-40 mg) were quantified using a hydroxylproline assay (Nanjing Jiancheng Bioengineering Institute, Jiangsu, China) according to the manufacturer's instructions. Hydroxyproline is a major component of the protein collagen. Using the alkaline hydrolysis method, hydroxyproline concentration is reflected as a colorimetric $(550 \mathrm{~nm})$ product that can be read with a spectrophotometer (Epoch2 microplate reader, BioTek, Vermont, USA), and then calculated using a standard formula according to the manufacturer's protocol.

Histopathology of mice lung tissue 
Mice were sacrificed using sodium pentobarbital anesthesia and cervical dislocation on day 21 or day 28 and the lung tissue was collected. Formalin-fixed and paraffin-embedded mice lung tissue was sectioned and mounted on adhesion microscope slides for staining with hematoxylin and eosin or Masson's trichrome according to established protocols. The histopathological scoring for inflammation and fibrosis were performed by two experienced pathologists. The score standards were determined according to the Ashcroft score on lung histology [23].

Quantitative Reverse Transcription Polymerase Chain Reaction (qRT-PCR)

Total RNA was extracted by using the EZgene ${ }^{T M}$ Tissue RNA Miniprep Kit (BIOMIGA, San Diego, CA, USA) according to the manufacturer's instructions. The ReverTra Ace qPCR RT Kit (TOYOBO, Osaka, Japan) was used for reverse transcription of RNA to CDNA. Quantitative real-time PCR was carried out using the ABI7500 system (Applied Biosystems). Primers for ATP6, CHOP, and $\beta$-actin (housekeeping gene) were obtained from Sangon Biotech (Shanghai, China) and their sequences are listed in Table 1. The PCR reaction was performed using the Thunderbird SYBR qPCR Mix Kit (TOYOBO, Osaka, Japan). The comparative cycle time (CT) method was used to determine the relative mRNA expression levels. The fold changes were calculated using the $2^{-} \Delta \triangle C T$ method normalized to the housekeeping gene $\beta$-actin.

Western blot

Mice lung tissue was homogenized and the total protein extracted using the radioimmunoprecipitation assay (RIPA) lysis buffer (Solarbio, Shanghai, China). The lysate was centrifuged and the supernatant was collected. The bicinchoninic acid (BCA) Protein Assay Kit (Biotechwell, Shanghai, China) was used to determine the protein concentration. For the analysis of ATF6 and CHOP protein expression, $15-20 \mu \mathrm{g}$ of lysates were separated on 10-12\% SDS-PAGE Tris Bis gels and then transferred to nitrocellulose membranes (Millipore, Billerica, MA, USA). The membranes were incubated with $5 \% \mathrm{w} / \mathrm{v}$ bovine serum albumin in tris-buffered saline with Tween 20 (TBST; Sigma-Aldrich) to block non-specific binding for $2 \mathrm{~h}$ at room temperature. After three washes, the membranes were incubated with the following primary antibodies: anti-ATF6 Ab (1:1000, Abcam, Cambridge, USA), anti-CHOP Ab (1:1000, Cell Signaling), or anti$\beta$-actin (1:2000, Arigo Biolaboratories, Shanghai, China) overnight at $4^{\circ} \mathrm{C}$. The membranes were subsequently incubated with horseradish peroxidase-labeled secondary antibodies (1:2000, Huabio, Hangzhou, China) for $1 \mathrm{~h}$ at room temperature. The protein bands were visualized using the ChemiDoc ${ }^{\mathrm{TM}}$ XRS+ System (Bio-Rad, Hercules, CA, USA). Protein expression levels were determined based on the relative fluorescence intensity of the protein band and normalized to the housekeeping protein $\beta$-actin. Image analysis was carried out using Image Lab ${ }^{\text {TM }}$ Software (Bio-Rad, Hercules, CA, USA).

Statistical analysis

All data were presented as the mean \pm standard error (SEM). Comparison of continuous variables was carried out using the unpaired Student's t-test for two groups or one-way analysis of variance (ANOVA) with Bonferroni's post hoc analysis for multiple groups. Survival data for the mouse model was analyzed using Kaplan-Meier survival curves and log-rank test. $p$ values $\leq 0.05$ were considered statistically 
significant. All statistical analyses were performed using GraphPad Prism 5.0 (GraphPad Software, San Diego, CA).

Table 1 Primers used in quantitative real-time PCR assays.

\begin{tabular}{lll} 
Genes & Forward primers $\left(5^{\prime} \rightarrow 3^{\prime}\right)$ & Reverse primers $\left(5^{\prime} \rightarrow 3^{\prime}\right)$ \\
\hline ATF6 & CGGTCCACAGACTCGTGTTC & GCTGTCGCCATATAAGGAAAGG \\
\hline CHOP & CTGGAAGCCTGGTATGAGGAT & CAGGGTCAAGAGTAGTGAAGGT \\
\hline -Actin & GGCTGTATTCCCCTCCATCG & CCAGTTGGTAACAATGCCATGT
\end{tabular}

\section{Results}

\section{IPF patients had increased expression of ATF6 and CHOP in lung}

The appearance of lung tissue from the IPF patients was heterogeneous upon histological examination. The pattern of usual interstitial pneumonia was a key histopathologic feature. Patchy fibrotic areas were mainly located in the peripheral zones of secondary lobules adjacent to normal-looking parenchyma. Fibroblastic foci were scattered within areas of dense collagen deposition, together with honeycomb changes in the subpleural region.

Immunohistochemistry revealed that ATF6 and CHOP protein were mainly present in fibroblasts or myofibroblasts in the fibroblastic foci. The percentage of ATF6 and CHOP-positive cells was significantly higher in the lungs of IPF patients (ATF6 median, 84\% [range, 80\%-90\%]; CHOP 74\% [65\%-80\%]) than in those from healthy controls (ATF6 13\% [5\%-25\%]; CHOP 7.5\% [5\%-25\%]) (all P<0.01) (Fig. 2).

\section{4-PBA attenuated pulmonary fibrosis in BLM-treated mice}

The presence of pulmonary fibrosis was verified in BLM-treated mice by hematoxylin and eosin staining and Masson's trichrome staining of the lung tissue. The acute lung injury (ALI) score and fibrosis score were determined to estimate the severity of pulmonary fibrosis. The fibrosis scores (day21 median, 0.8 [range, 1-2]; day28 1.9 [1-3]) were significantly lower in the BLM-treated mice who received 4-PBA treatment than those in the BLM-only group (day21 3 [2-5]; day28 5.4 [3-7]) $(P<0.01)$. The results showed that BLM-treated mice who also received 4-PBA treatment exhibited decreased collagen deposition on day 21 and day 28 compared to the BLM-only group (Fig. 3C, D). The ALI scores (day21 median, 3 [range, 2-5]; day28 1.8 [1-3]) were also significantly lower in the BLM-treated mice who received 4-PBA treatment than those in the BLM-only group (day21 median 5.3, [3-8]; day28 3.5, [2-6]) $(\mathrm{P}<0.01)$. 4-PBA also dramatically attenuated the lung damage induced by BLM on day 21 and day 28 (Fig. 3A, B).

\section{4-PBA increased survival of mice with BLM-induced pulmonary fibrosis}


Survival analysis showed that the BLM-treated mice who also received 4-PBA treatment from day 15 to day 28 showed a higher survival rate than the BLM-only group (Fig. 4). Hydroxyproline, which is a surrogate marker for collagen deposition, increased following BLM treatment (day21 median, 1.09 [range, 0.90-1.34]; day28 1.69 [1.33-1.95]), and was significantly decreased by 4-PBA (day21 0.60 [0.49-0.72]; day28 0.74 [0.58-0.95]) $\quad($ day21 $\mathrm{P}<0.05$; day28 $\mathrm{P}<0.01$ ) (Fig. 5). These results strongly suggested that 4PBA had a positive effect on the survival of mice with BLM-induced pulmonary fibrosis and markedly reduced collagen deposition.

\section{4-PBA improved lung function of mice with BLM-induced pulmonary fibrosis}

To determine whether normal pulmonary function in mice was impaired by BLM and improved by 4-PBA, the mice were placed in a plethysmograph and the lung function parameters FVC, FEV50, and Cydn were measured. Compared to the control mice (FVC median, 1.35 [range, 1.32-1.39]; FEV50 1.05 [0.98-1.09]; cydn 0.036 [0.031- 0.039]), significant decreases in FVC (day21 0.88 [0.70-1.07], $P<0.01$; day28 0.50 [0.380.68], $\mathrm{P}<0.01$ ), FEV50 (day21 0.79 [0.69-1.02], $\mathrm{P}<0.01$; day28 0.40 [0.21-0.70], $\mathrm{P}<0.01$;), and Cydn (day21 0.019 [0.014-0.027] , $P<0.01$; day28 0.013 [0.010-0.022] , $P<0.01$ ), were seen in the BLM-treated mice on day 21 and day 28. Notably, increases in FVC (day21 1.15 [0.94-1.33], $P<0.01$; day28 0.99 [0.94-1.11], $\mathrm{P}<0.05$ ), FEV50 (day21 0.90 [0.78-1.02], not significant; day28 0.81 [0.64-0.94], $\mathrm{P}<0.05$ ), and Cydn (day21 0.033 [0.031-0.035], $P<0.05$; day28 0.027 [0.020-0.036], $P<0.01$ ) were markedly observed after 4-PBA treatment in mice with BLM-induced pulmonary fibrosis on day 21 and day 28 (Fig. 6A, B, and C).

\section{4-PBA decreased the level of ER stress and prevented the expression of ATF6 and CHOP}

To evaluate the potential effect of 4-PBA in ER stress, the mRNA and protein expression levels for the ER stress markers ATF6 and CHOP in mice lung tissue were analyzed. Mice from the BLM group expressed higher levels of both ATF6 and CHOP mRNA and protein in their lungs than the control mice. 4-PBA reduced ATF6 and CHOP mRNA and protein expression levels after 1 week of treatment (Fig. 7A, B, and C). It should be noted that both decreases were more significant after 2 weeks of 4-PBA treatment (Fig. 7D, E, and F).

\section{Discussion}

IPF is a devastating disorder with a high mortality rate [24] and unknown cause. To date, there is no effective pharmacological treatment. Recently, a growing number of studies have demonstrated that ER stress plays an important role in IPF [2]. In 2001, Nogee et al. [11] reported that an infant with interstitial lung disease born to a woman who had desquamative interstitial pneumonia had a mutation in the SFTPC gene. Subsequently, Thomas et al. [25] linked a different SFTPC mutation that led to the misfolding and accumulation of the surfactant protein $C$ precursor protein in the ER with familial pulmonary fibrosis (FPF). In 2008, Lawson et al. [26] found that ER stress and UPR activation were not only present in FPF patients with SFTPC mutations, but also in FPF patients without SFTPC mutations as well as in patients with sporadic IPF. Baek et al. [12] identified an upregulation in the expression of UPR markers such as GRP78 as well as CHOP and ATF6 in lung tissues from both BLM-treated mice and IPF 
patients. The present study has demonstrated that the expression levels of ATF6 and CHOP were increased both in IPF patients and in a BLM-induced mouse model of pulmonary fibrosis, which is consistent with the results in the published literature. Moreover, 4-PBA effectively inhibited the expression of ER stress markers and thereby alleviated fibrosis progression in mice.

4-PBA has been shown to exert protective effects on fibrotic processes [27]. Rishikof et al. [28] found that 4-PBA could reduce type I collagen deposition in human lung fibroblasts. In the transforming growth factor (TGF)- $\beta 1$-induced mice and human fibroblasts, 4-PBA was shown to reduce the expression of UPR markers such as GRP78, X-box binding protein 1, and ATF6a, along with the levels of myofibroblast markers such as a-SMA and type I collagen, which suggested that 4-PBA was able to attenuate ER stress and inhibit the myofibroblastic differentiation of fibroblasts in IPF [12]. In RLE-6TN cells, 4-PBA was found to suppress TGF- $\beta 1$-induced epithelial-mesenchymal transition via the inhibition of ER stress, which in turn blocked the progression of IPF [29]. In the present study, we observed that 4-PBA reduced collagen deposition in BLM-induced pulmonary fibrosis. We also found that 4-PBA improved the lung function and survival rate. Moreover, our data showed that the expression of CHOP and ATF6 mRNA and protein decreased with 4-PBA treatment, which suggested that 4-PBA alleviated BLM-induced pulmonary fibrosis via attenuation of the ATF6 pathway. However, ER stress is a ubiquitous protection mechanism. Further study is required to determine the optimal time and course of 4-PBA treatment for IPF.

\section{Conclusion}

Taken together, the present study demonstrated that 4-PBA protected mice from BLM-induced pulmonary fibrosis via the inhibition of ER stress. This may provide new insights for the development of future therapies for IPF.

\section{Abbreviations}


4-PBA 4-phenylbutyric acid

ER endoplasmic reticulum

UPR unfolded protein response

IPF idiopathic pulmonary fibrosis

BLM bleomycin

ATF6 activating transcription factor 6

CHOP C/EBP Homologous Protein

IRE1a inositol-requiring enzyme 1 alpha

PERK PKR-like ER kinase

BiP immunoglobulin heavy-chain-binding protein

SFTPC surfactant protein C

FVC forced vital volume

FEV50 forced expiratory volume in 50 milliseconds

Cdyn dynamic compliance

qRT- Quantitative Reverse Transcription Polymerase Chain Reaction Polymerase Chain

PCR Reaction (qRT-PCR) Polymerase Chain Reaction (qRT-PCR)

Chain Reaction (qRT-PCR)

CT comparative cycle time

SEM standard error

ANOVA one-way analysis of variance

ALI acute lung injury

FPF familial pulmonary fibrosis

TGF transforming growth factor

\section{Declarations}

Ethics approval and consent to participate: The study was approved by the Ethics Committee of Shanghai Pulmonary Hospital (Approval No: 2014FK04, Approved date: February $25^{\text {th }}$, 2014). Written informed consent was obtained from all participants. The study protocol was approved by the Institutional Animal Care and Use Committee at Soochow University (Approval No.: K18-028). 
Consent for publication: All authors approved the final manuscript.

Availability of data and material『All data generated or analyzed during this study are included in this published article and its supplementary information files.

Competing interests: No potential conflicts of interest were disclosed.

Funding: Supported by grants from the National Natural Science Foundation of China (grant numbers: 81900058) and Changzhou Health Commission (grant numbers: ZD201801). The funders had no role in the design of the study and collection, analysis, and interpretation of data and in writing the manuscript.

Authors' contributions: Experimental design: H.Q., J.Z., Y.Y., and D.Y.C.; Data acquisition and analysis: Y.Y., D.Y.C., H.B., Q.H., Y.Q.G., Q.D.Z., J.Z., and H.Q.; Writing the manuscript: Y.Y., D.Y.C., J.Z., and H.Q.. All authors have read and approved the manuscript.

Acknowledgements $\triangle$ The authors would like to thank Dr. Hui Wang, Dr. Yan Tan, Dr. Yong-qiang Shi (Department of Pathology, The First People's Hospital of Changzhou, The Third Affiliated Hospital of Soochow University) for the pathologic evaluation of our histology specimens. The authors thank Professor Robert P. Baughman and Medjaden BioscienceLimited for editing and proofreading the manuscript.

\section{References}

1. Loomis-King H, Flaherty KR, Moore BB: Pathogenesis, current treatments and future directions for idiopathic pulmonary fibrosis. Curr Opin Pharmacol 2013, 13(3):377-385.

2. Lawson WE CD, Degryse AL,Tanjore H,Polosukhin VV,Xu XC,Newcomb DC,Jones BR,Roldan J,Lane KB,Morrisey EE: Endoplasmic reticulum stress enhances fibrotic remodeling in the lungs. Proc NatI Acad Sci U S A 2011, 108(26):10562-10567.

3. Korfei M, Ruppert C, Mahavadi P, Henneke I, Markart P, Koch M, Lang G, Fink L, Bohle RM, Seeger W et al: Epithelial endoplasmic reticulum stress and apoptosis in sporadic idiopathic pulmonary fibrosis. American journal of respiratory and critical care medicine 2008, 178(8):838-846.

4. Zhang L, YW, NSP, GW, XX, QG, WX, C-YW, PY, BR: 2017 Zhang L Endoplasmic reticulum stress a new wrestler in the pathogenesis of idiopathic pulmonary fibrosis. Am J Transl Res 2017.

5. Lenna S, Han R, Trojanowska M: Endoplasmic reticulum stress and endothelial dysfunction. IUBMB life 2014, 66(8):530-537.

6. Grootjans J, Kaser A, Kaufman RJ, Blumberg RS: The unfolded protein response in immunity and inflammation. Nature reviews Immunology 2016, 16(8):469-484.

7. Harikrishna Tanjore TSB, William E. Lawson: Emerging evidence for endoplasmic reticulum stress in the pathogenesis of idiopathic pulmonary fibrosis. Am J Physiol Lung Cell Mol Physiol 2012.

8. Kolb PS, Ayaub EA, Zhou W, Yum V, Dickhout JG, Ask K: The therapeutic effects of 4-phenylbutyric acid in maintaining proteostasis. The international journal of biochemistry \& cell biology 2015, 


\section{1:45-52.}

9. Ayala P, Montenegro J, Vivar R, Letelier A, Urroz PA, Copaja M, Pivet D, Humeres C, Troncoso R, Vicencio JM et al: Attenuation of endoplasmic reticulum stress using the chemical chaperone 4phenylbutyric acid prevents cardiac fibrosis induced by isoproterenol. Experimental and molecular pathology 2012, 92(1):97-104.

10. Mercado G, Castillo V, Vidal R, Hetz C: ER proteostasis disturbances in Parkinson's disease: novel insights. Frontiers in aging neuroscience 2015, 7:39.

11. Nogee LM, Dunbar, A. E., Wert, S. E., Askin, F., Hamvas, A., \& Whitsett, J. A.: A mutation in the surfactant protein $\mathrm{C}$ gene associated with familial interstitial lung disease. New England Journal of Medicine 2001, 344(8):573-579.

12. Baek HA, Kim DS, Park HS, Jang KY, Kang MJ, Lee DG, Moon WS, Chae HJ, Chung MJ: Involvement of endoplasmic reticulum stress in myofibroblastic differentiation of lung fibroblasts. American journal of respiratory cell and molecular biology 2012, 46(6):731-739.

13. Xu P, Yao Y, Zhou J: Particulate matter with a diameter of </=2.5 mum induces and enhances bleomycin-induced pulmonary fibrosis by stimulating endoplasmic reticulum stress in rat. Biochemistry and cell biology = Biochimie et biologie cellulaire 2019, 97(4):357-363.

14. Qiu H, Weng D, Chen T, Shen L, Chen SS, Wei YR, Wu Q, Zhao MM, Li QH, Hu Y et al: Stimulator of Interferon Genes Deficiency in Acute Exacerbation of Idiopathic Pulmonary Fibrosis. Frontiers in immunology 2017, 8:1756.

15. Chen T, Qiu H, Zhao M-M, Chen S-S, Wu Q, Zhou N-Y, Lu L-Q, Song J-C, Tang D-L, Weng D et al: IL-17A contributes to HSV1 infection-induced acute lung injury in a mouse model of pulmonary fibrosis. Journal of cellular and molecular medicine 2019, 23(2):908-919.

16. Lichter-Konecki U, Diaz GA, Merritt JL, Feigenbaum A, Jomphe C, Marier JF, Beliveau M, Mauney J, Dickinson K, Martinez A et al: Ammonia control in children with urea cycle disorders (UCDs); Phase 2 comparison of sodium phenylbutyrate and glycerol phenylbutyrate. Molecular Genetics and Metabolism 2011, 103(4):323-329.

17. Ricobaraza A C-TM: Long-term phenylbutyrate administration prevents memory deficits in Tg2576 mice by decreasing Abeta. Front Biosci 2011, 3:1375-1384.

18. Chatziantoniou C, Carlisle RE, Brimble E, Werner KE, Cruz GL, Ask K, Ingram AJ, Dickhout JG: 4Phenylbutyrate Inhibits Tunicamycin-Induced Acute Kidney Injury via CHOP/GADD153 Repression. PLOS ONE 2014, 9(1):e84663.

19. Luo T, Chen B, Wang X: 4-PBA prevents pressure overload-induced myocardial hypertrophy and interstitial fibrosis by attenuating endoplasmic reticulum stress. Chemico-Biological Interactions 2015, 242:99-106.

20. Koyama M, Furuhashi M, Ishimura S, Mita T, Fuseya T, Okazaki Y, Yoshida H, Tsuchihashi K, Miura T: Reduction of endoplasmic reticulum stress by 4-phenylbutyric acid prevents the development of hypoxia-induced pulmonary arterial hypertension. American journal of physiology Heart and circulatory physiology 2014, 306(9):H1314-1323. 
21. Lee HY, Marahatta A, Bhandary B, Kim HR, Chae HJ: 4-Phenylbutyric acid regulates CCl4-induced acute hepatic dyslipidemia in a mouse model: A mechanism-based PK/PD study. European journal of pharmacology 2016, 777:104-112.

22. Sisson TH, Hansen JM, Shah M, Hanson KE, Du M, Ling T, Simon RH, Christensen PJ: Expression of the reverse tetracycline-transactivator gene causes emphysema-like changes in mice. Am J Respir Cell Mol Biol 2006, 34(5):552-560.

23. Ashcroft T SJ: Simple method of estimating severity of pulmonary fibrosis on a numerical scale. $J$ Clin Pathol 1988, 41(4):467-470.

24. Snyder L, Neely ML, Hellkamp AS, O'Brien E, de Andrade J, Conoscenti CS, Leonard T, Bender S, Gulati $M$, Culver DA et al: Predictors of death or lung transplant after a diagnosis of idiopathic pulmonary fibrosis: insights from the IPF-PRO Registry. Respiratory research 2019, 20(1):105.

25. Thomas AQ, Lane K, Phillips J, 3rd, Prince M, Markin C, Speer M, Schwartz DA, Gaddipati R, Marney $\mathrm{A}$, Johnson $\mathrm{J}$ et al: Heterozygosity for a surfactant protein $\mathrm{C}$ gene mutation associated with usual interstitial pneumonitis and cellular nonspecific interstitial pneumonitis in one kindred. American journal of respiratory and critical care medicine 2002, 165(9):1322-1328.

26. Lawson WE CP, Polosukhin VV,Roldan J,Cheng DS,Lane KB,Blackwell TR,Xu C,Markin C,Ware LB,Miller GG: Endoplasmic reticulum stress in alveolar epithelial cells is prominent in IPF: association with altered surfactant protein processing and herpesvirus infection. Am J Physiol Lung Cell Mol Physiol 2008, 294(6):L1119-1126.

27. Liu SH, Yang CC, Chan DC, Wu CT, Chen LP, Huang JW, Hung KY, Chiang CK: Chemical chaperon 4phenylbutyrate protects against the endoplasmic reticulum stress-mediated renal fibrosis in vivo and in vitro. Oncotarget 2016, 7(16):22116-22127.

28. Rishikof DC, Ricupero DA, Liu H, Goldstein RH: Phenylbutyrate decreases type I collagen production in human lung fibroblasts. Journal of Cellular Biochemistry 2004, 91(4):740-748.

29. Mo XT, Zhou WC, Cui WH, Li DL, Li LC, Xu L, Zhao P, Gao J: Inositol-requiring protein 1 - X-box-binding protein 1 pathway promotes epithelial-mesenchymal transition via mediating snail expression in pulmonary fibrosis. The international journal of biochemistry \& cell biology 2015, 65:230-238.

\section{Figures}


- Evaluation of pulmonary damage

- Determination of lung function test

- Assessment ERS markers in lung tissue

- Estimation of 4-PBA role

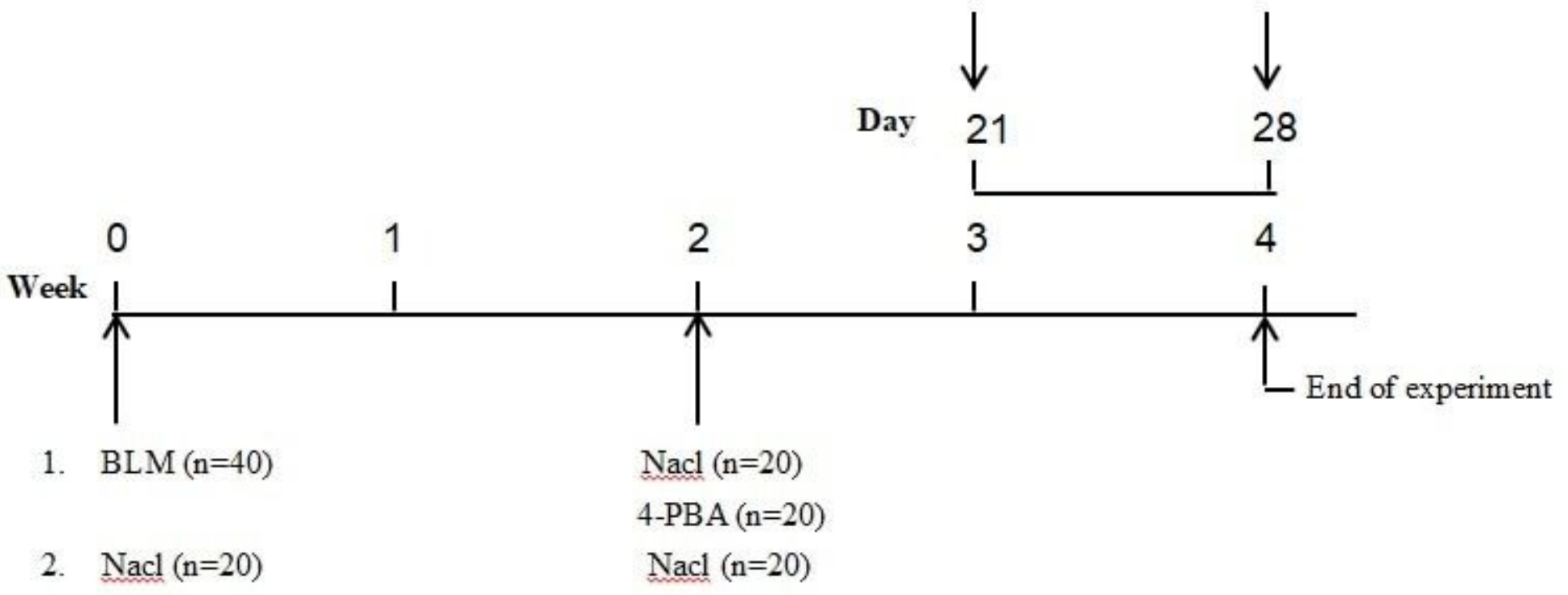

Figure 1

Mouse modeling workflow. Abbreviations: BLM: bleomycin; Nacl: Sodium chloride; 4-PBA: 4-phenylbutyric acid; ERS: endoplasmic reticulum stress.

A
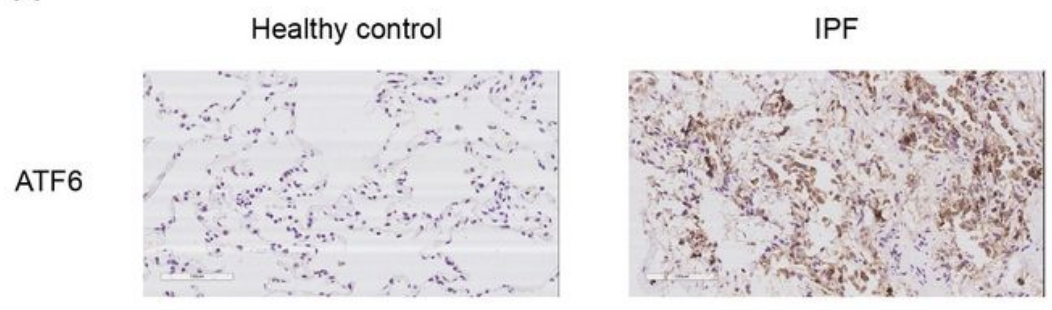

C

Healthy control

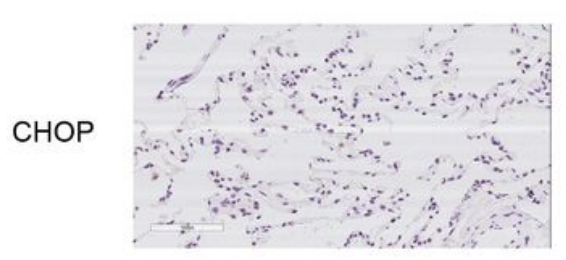

IPF

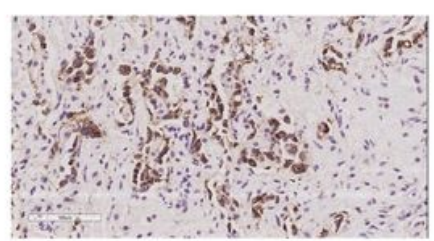

B

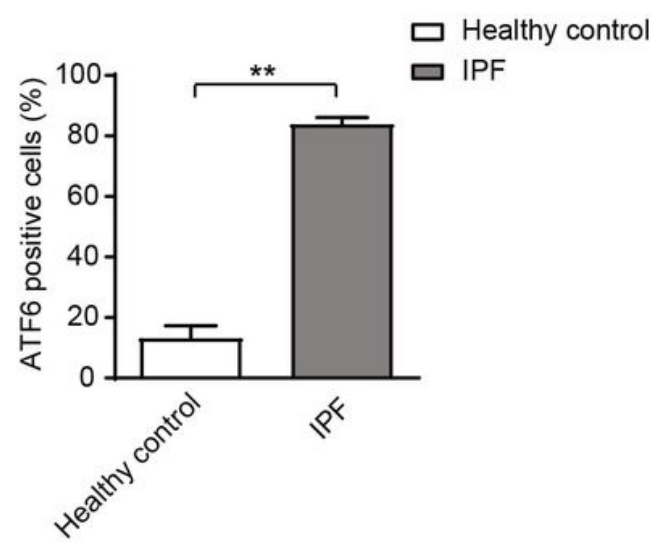

D

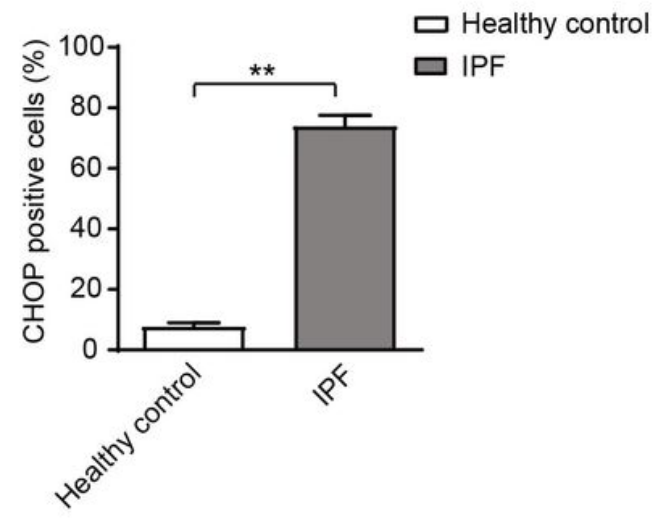


Figure 2

Expression of ATF6 and CHOP protein in lung tissues from IPF patients and control A. Representative immunohistochemical staining for ATF6 in lung tissues from patients with IPF $(n=4)$ and healthy controls $(n=4)$. B. The percentages of ATF6-positive cells. C. Representative immunohistochemical staining for $\mathrm{CHOP}$ in lung tissues from patients with IPF $(n=4)$ and healthy controls $(n=4)$. D. The percentages of CHOP-positive cells. The expression levels of ATF6 and CHOP were significantly increased in lung tissues from IPF patients compared to those in healthy controls. ATF6 and CHOP were detected in fibroblasts and myofibroblasts in fibroblastic focus (brown staining). (Hematoxylin and eosin staining: 20x, LEICA SCN400, scale bar $=100 \mu \mathrm{m})$. Abbreviations: ATF6: activating transcription factor 6; CHOP: C/EBP Homologous Protein; IPF: idiopathic pulmonary fibrosis.

A
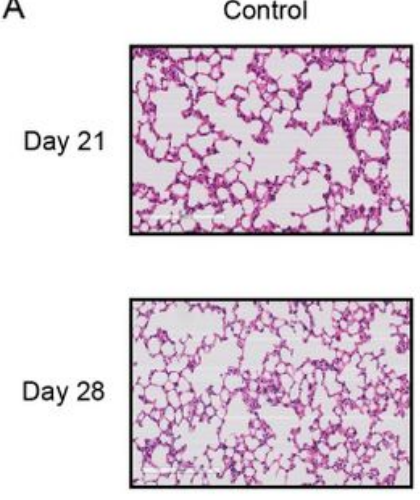

C
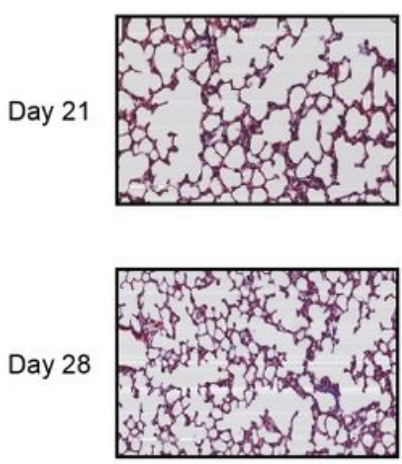

BLM
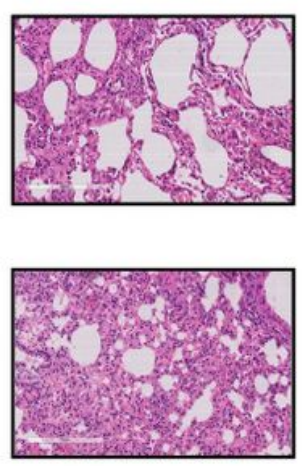

BLM
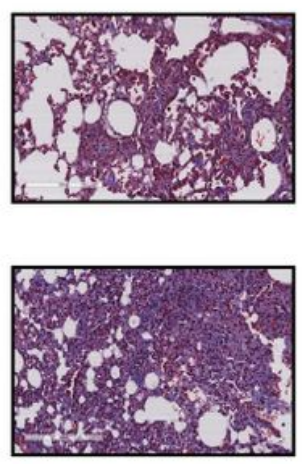

BLM+4-PBA
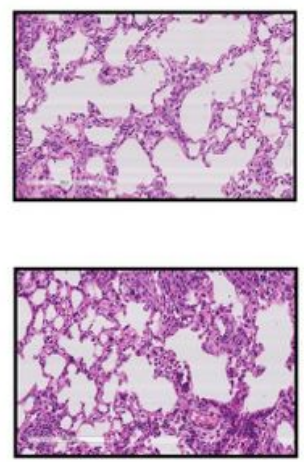

BLM+4-PBA
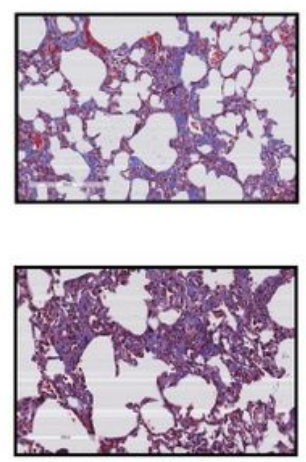

B

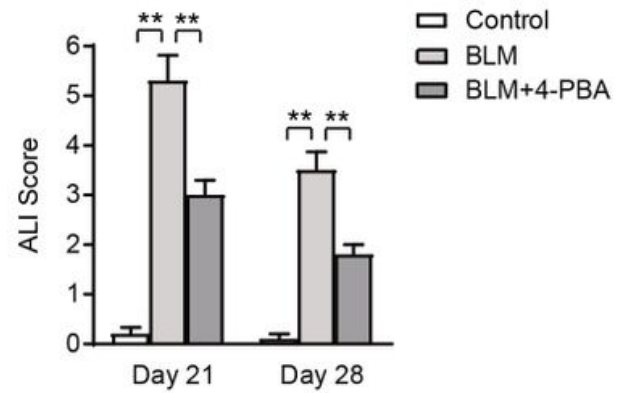

D

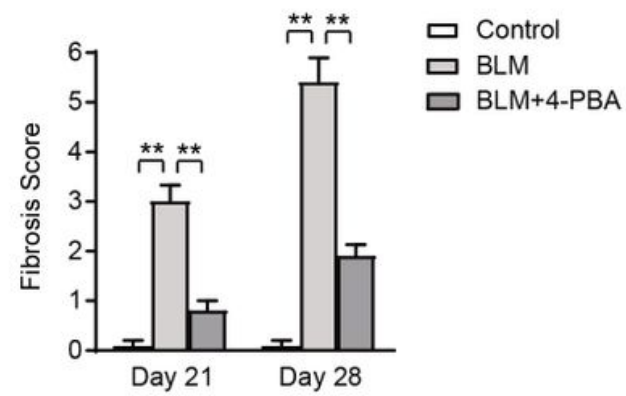

\section{Figure 3}

Histopathology of the mouse lung tissues A. Representative images of lung sections with hematoxylin and eosin staining $(20 x$, LEICA SCN400, scale bar $=200 \mu \mathrm{m})$. B. ALI scores for the lung tissues. C. Representative images of the lung sections with Masson's trichrome staining (20x, LEICA SCN400, scale bar $=200 \mu \mathrm{m})$. D. Fibrosis scores for the lung tissues. Data are presented as mean $\pm S E M, \star \star p<0.01$. Abbreviations: BLM: bleomycin; 4-PBA: 4-phenylbutyric acid; ALI: acute lung injury. 


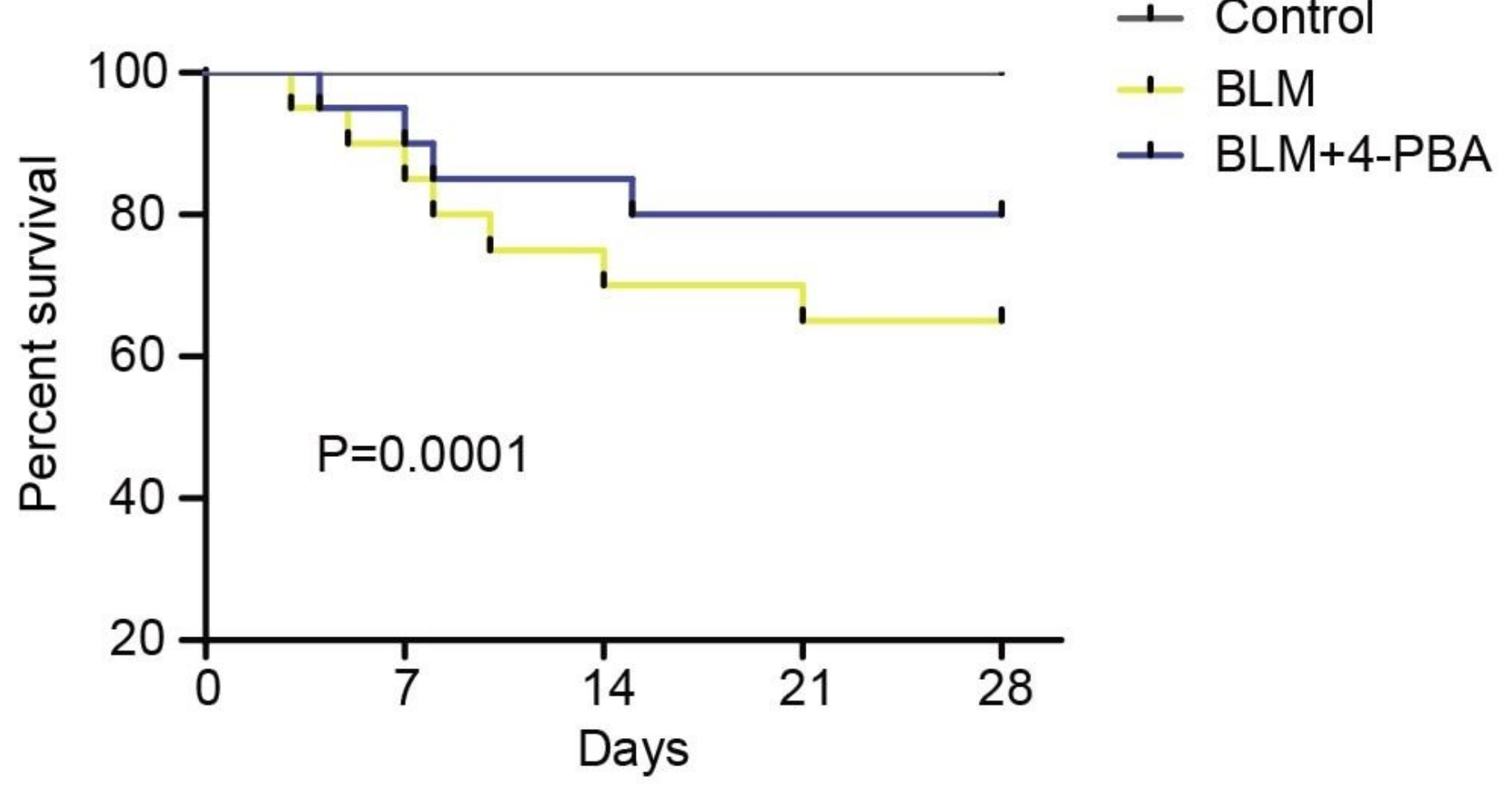

Figure 4

Mouse survival Kaplan-Meier analysis of mouse survival $(n=20)$. Abbreviations: BLM: bleomycin; 4-PBA: 4-phenylbutyric acid.

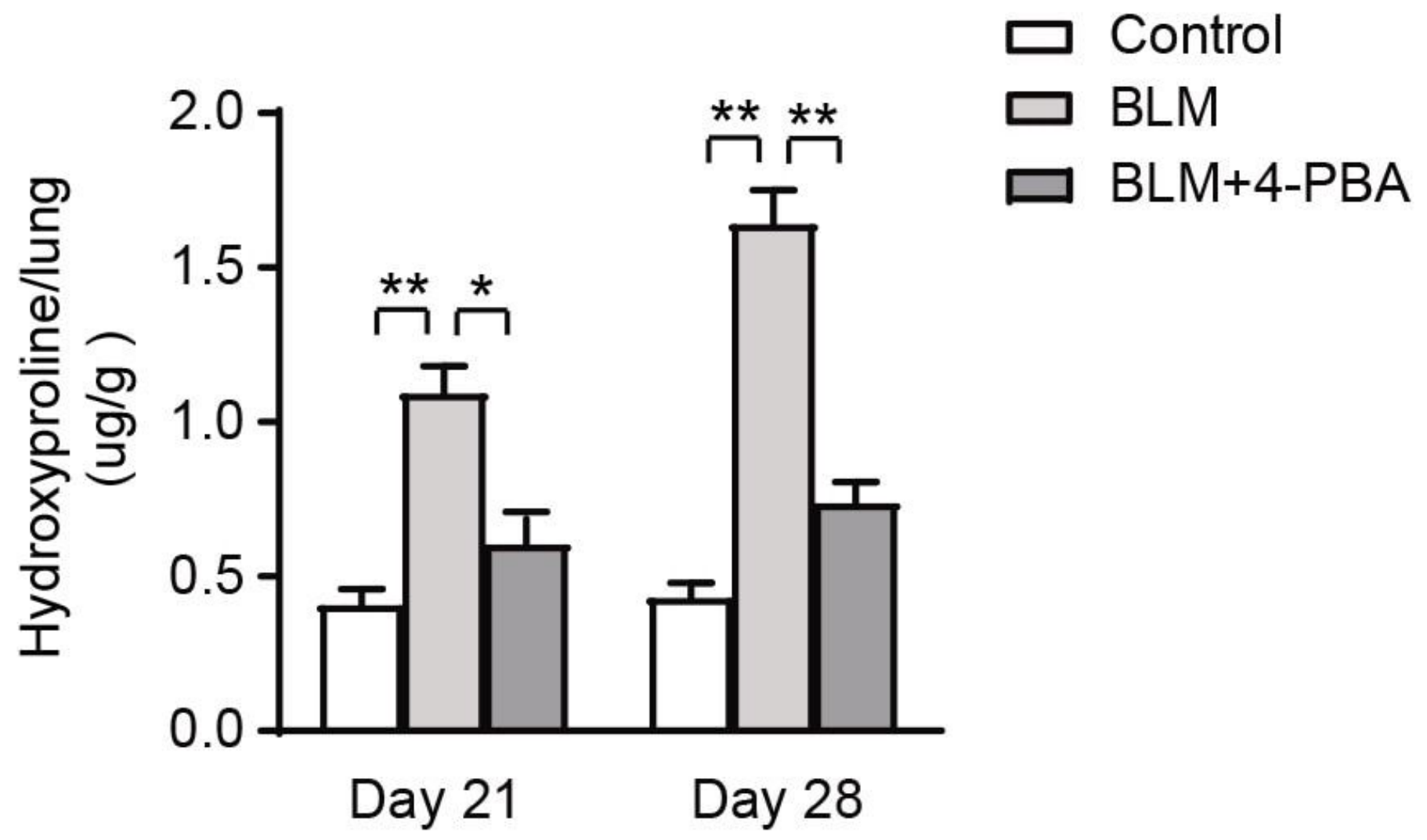

Figure 5 
The hydroxyproline content of mouse lungs $(n=20)$ Data are presented as mean $\pm S E M,{ }^{\star} p<0.05$; ${ }^{*} p$ $<0.01$. Abbreviation: BLM: bleomycin; 4-PBA: 4-phenylbutyric acid.

A

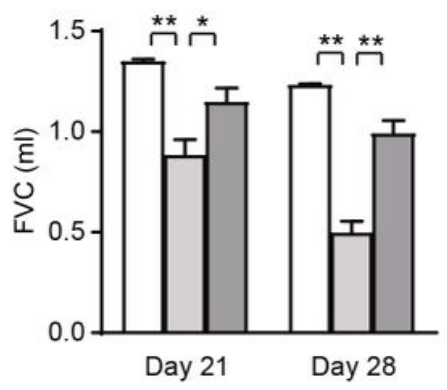

B

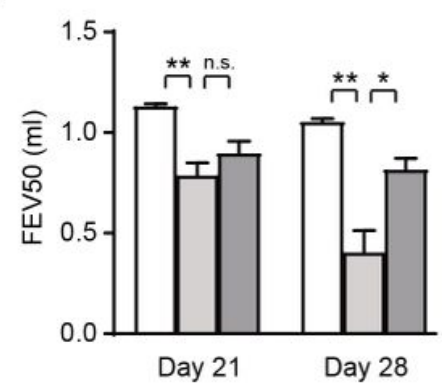

C

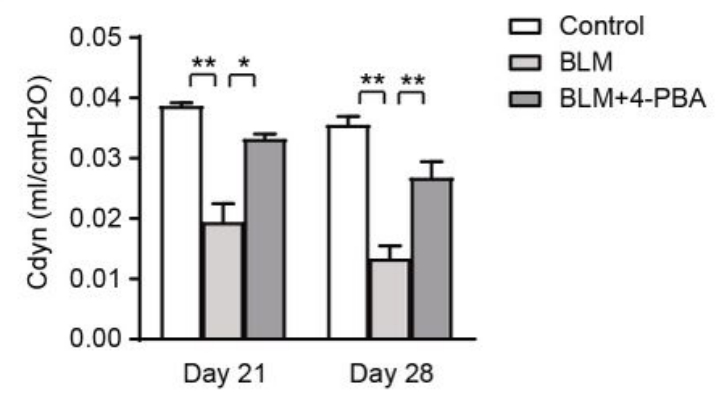

Figure 6

Pulmonary function of mice A. FVC of mice $(n=20)$. B. FEV50 of mice $(n=20)$. C. Cdyn of mice $(n=20)$. Data are presented as means $\pm S E M,{ }^{*} p<0.05 ;{ }^{* \star} p<0.01$; n. s., not significant. Abbreviation: BLM: bleomycin; 4-PBA: 4-phenylbutyric acid; FVC: forced vital volume; FEV50: forced expiratory volume in 50 milliseconds; Cdyn: dynamic compliance.

A

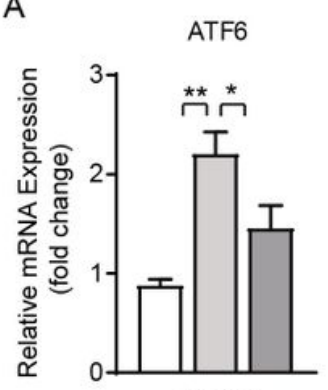

Day 21

D

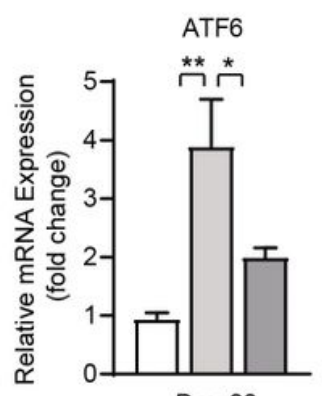

Day 28

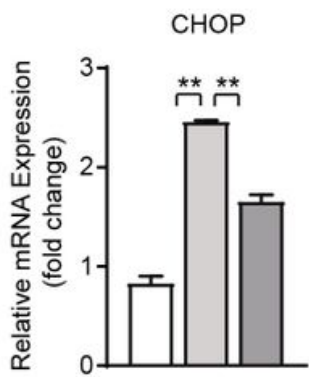

Day 21

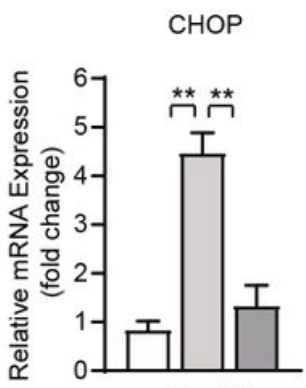

Day 28
B

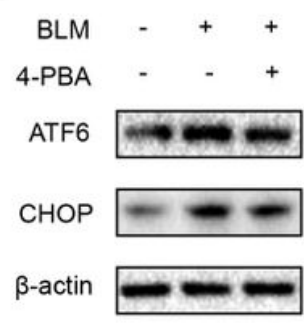

E

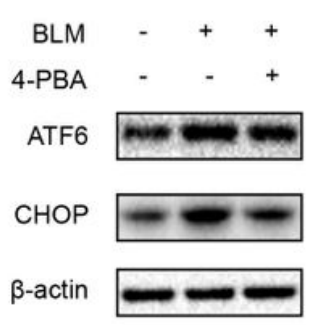

C

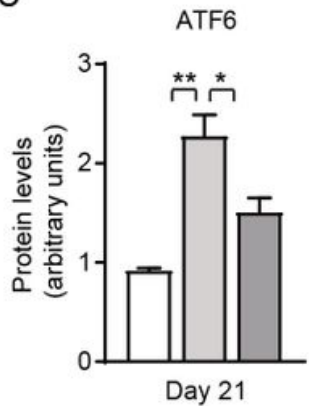

$\mathrm{F}$

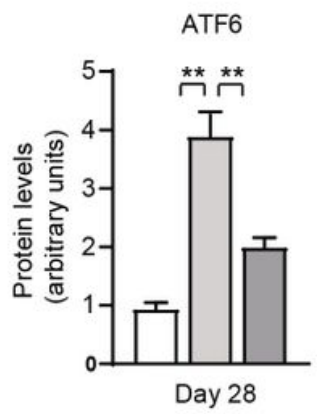

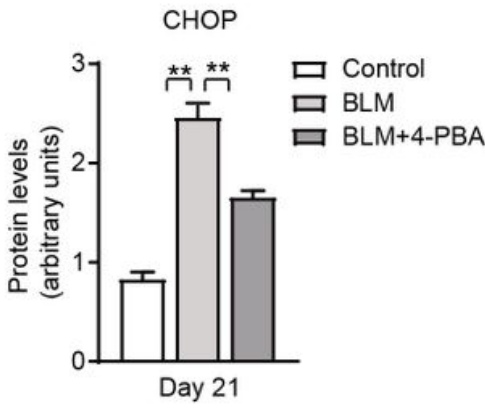

CHOP

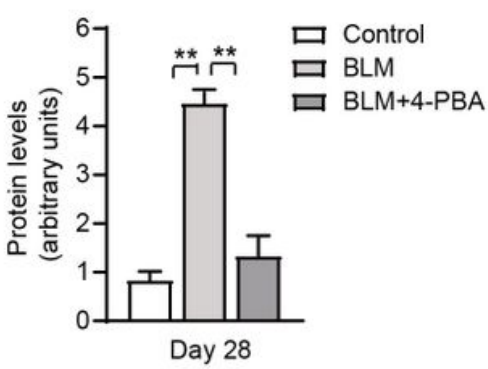

Figure 7

4-PBA decreased mRNA and protein expression of ER stress markers ATF6 and CHOP in mouse lungs A. mRNA expression of ATF6 and CHOP on day $21(n=10)$. B: Representative Western blot image of ATF6 and CHOP protein expression on day 21. C. Densitometry analysis of the Western blot bands on day 21 normalized to $\beta$-actin $(n=5)$. D. mRNA expression of ATF6 and CHOP on day $28(n=10)$. E. Representative Western blot image of ATF6 and CHOP protein expression on day 28. F. Densitometry analysis of the 
Western blot bands on day 28 normalized to $\beta$-actin $(n=5)$. Data are presented as mean $\pm S E M, * p<0.05$; $\star \star p<0.01$; n. s., not significant. 